\title{
THE SURFACE AREA PRESERVING MEAN CURVATURE FLOW *
}

\section{JAMES MCCOY ${ }^{\dagger}$}

Let $M_{0}$ be a compact, strictly convex hypersurface of dimension $n \geq 2$, without boundary, smoothly embedded in $\mathbb{R}^{n+1}$ and represented locally by some diffeomorphism $F_{0}: \mathbb{R}^{n} \supset U \rightarrow F_{0}(U) \subset M_{0} \subset \mathbb{R}^{n+1}$. Under the surface area preserving mean curvature flow, formulated by Pihan in $[\mathrm{P}]$, the family of maps $F_{t}=F(\cdot, t)$ evolves according to

$$
\begin{gathered}
\frac{\partial}{\partial t} F(x, t)=\{1-h(t) H(x, t)\} \nu(x, t), x \in U, 0 \leq t \leq T \leq \infty, \\
F(\cdot, 0)=F_{0},
\end{gathered}
$$

where $H$ is the mean curvature of $M_{t}=F_{t}(U), \nu$ is the outer unit normal to $M_{t}$ and

$$
h(t)=\frac{\int_{M_{t}} H d \mu_{t}}{\int_{M_{t}} H^{2} d \mu_{t}},
$$

where $d \mu_{t}$ is the surface area element on $M_{t}$. Pihan studied basic properties of this flow for general $n$ and showed that (1) has a unique solution for a short time. He also proved for $n=1$ that an initially closed, convex curve in the plane converges exponentially to a circle with the same length as the initial curve. Analogous to this result and those of Huisken in [Hu1] and [Hu2] for the mean curvature flow and the volume preserving mean curvature flow, we show here a similar result for the surface area preserving flow, when $n \geq 2$. I would like to thank my $\mathrm{PhD}$ supervisor Professor Klaus Ecker for introducing me to this topic and for his suggestions, advice and encouragement throughout the production of this work. I would also like to thank Professor Gerhard Huisken and Dr Ben Andrews for useful suggestions and Associate Professors John Stillwell and Alan Pryde and Drs Maria Athanassenas and Marty Ross for valuable discussions.

Recently in $[\mathrm{M}]$ we have generalised the results of [Hu2] and this paper, studying general 'mixed volume' preserving mean curvature flows, of which the volume preserving and surface area preserving mean curvature flows are special cases.

1. Notation, definitions and well-known facts. We adopt similar notation to Huisken in [Hu1] and [Hu2]. Let $M$ be an $n$-dimensional Riemannian manifold. Vectors on $M$ are denoted by $X=\left\{X^{i}\right\}$, covectors by $Y=\left\{Y_{i}\right\}$ and mixed tensors by $T=\left\{T_{j}^{i k}\right\}$. The induced metric and the second fundamental form on $M$ are denoted by $g=\left\{g_{i j}\right\}$ and $A=\left\{h_{i j}\right\}$ respectively. The surface area element of $M$ is given by

$$
\mu=\sqrt{\operatorname{det}\left(g_{i j}\right)},
$$

while the inner product of tensors $T=\left\{T_{k m}^{i}\right\}$ and $S=\left\{S_{k m}^{i}\right\}$ on $M$ is written as

$$
\left\langle T_{k m}^{i}, S_{k m}^{i}\right\rangle=g_{i j} g^{k l} g^{m n} T_{k m}^{i} S_{l n}^{j}=T_{k m}^{i} S_{i}{ }^{k m}
$$

${ }^{*}$ Received October 10, 2002; accepted for publication December 6, 2002. This work was completed while the author was supported by an Australian Postgraduate Award and a Monash Postgraduate Publications Award.

${ }^{\dagger}$ Centre for Mathematics and its Applications, Mathematical Sciences Institute, Australian National University, ACT 0200, Australia (James.McCoy@anu.edu.au). 
where $g^{i j}$ is the $(i, j)$ entry of the inverse of the matrix $\left(g_{i j}\right)$. Unless otherwise indicated we always sum over repeated indices from 1 to $n$. So for the norm of the tensor $T$,

$$
\left|T_{k m}^{i}\right|^{2}=|T|^{2}=\left\langle T_{k m}^{i}, T_{k m}^{i}\right\rangle
$$

(The notation $\langle\cdot, \cdot\rangle$ is also used to denote the ordinary scalar product of vectors in $\mathbb{R}^{n+1}$.) For traces of the second fundamental form, we write

- $H=g^{i j} h_{i j}=h_{i}^{i}$, the mean curvature of $M$,

- $|A|^{2}=g^{i j} g^{k l} h_{i k} h_{j l}=h_{k}^{j} h_{j}{ }^{k}$, the norm of the second fundamental form on $M$,

- $C=g^{i j} g^{k l} g^{m n} h_{i k} h_{j m} h_{l n}=h_{k}^{j} h_{j}{ }^{n} h_{n}^{k}$,

- $Z=H C-|A|^{4}$ where $|A|^{4}:=\left(|A|^{2}\right)^{2}$.

If $F: U \subset \mathbb{R}^{n} \rightarrow \mathbb{R}^{n+1}$ smoothly embeds $M$ in $\mathbb{R}^{n+1}$, then

$$
g_{i j}(x)=\left\langle\frac{\partial F}{\partial x_{i}}(x), \frac{\partial F}{\partial x_{j}}(x)\right\rangle \text { and } h_{i j}(x)=\left\langle\frac{\partial F}{\partial x_{i}}(x), \frac{\partial \nu}{\partial x_{j}}(x)\right\rangle
$$

respectively, for $x \in U$, where $\nu(x)$ is the outer unit normal to $M$ at $F(x)$. The matrix of the Weingarten map of $M$ is then

$$
\left(h_{j}^{i}(x)\right)=\left(g^{i j}(x)\right)\left(h_{i j}(x)\right) .
$$

The eigenvalues of this matrix are the principal curvatures of $M . M$ is strictly convex if all the eigenvalues of $\left(h_{j}^{i}\right)$ are strictly positive.

The induced connection on $M$ is given via the Christoffel symbols,

$$
\Gamma_{i j}^{k}=\frac{1}{2} g^{k l}\left(\frac{\partial}{\partial x_{i}} g_{j l}+\frac{\partial}{\partial x_{j}} g_{i l}-\frac{\partial}{\partial x_{l}} g_{i j}\right)
$$

so the covariant derivative of a vector $X$ is

$$
\nabla_{j} X^{i}=\frac{\partial}{\partial x_{j}} X^{i}+\Gamma_{k j}^{i} X^{k}
$$

The covariant derivative of a tensor $T$ is denoted $\nabla T=\left\{\nabla_{l} T_{j}^{i k}\right\}$. The Laplacian of $T$ is

$$
\Delta T_{j}^{i k}=\nabla^{m} \nabla_{m} T_{j}^{i k}
$$

The Riemannian curvature tensor on $M$ may be given through the Gauss equations

$$
R_{i j k l}=h_{i k} h_{j l}-h_{i l} h_{j k}
$$

Then the formulas for interchanging second covariant derivatives are

$$
\nabla_{i} \nabla_{j} X^{h}-\nabla_{j} \nabla_{i} X^{h}=R_{i j k}^{h} X^{k}
$$

and

$$
\nabla_{j} \nabla_{i} Y_{k}-\nabla_{j} \nabla_{i} Y_{k}=R_{i j k}^{m} Y_{m}
$$


LEMMA 1.1.

i) $\nabla_{i} h_{j k}=\nabla_{j} h_{k i}=\nabla_{k} h_{i j}$ (the Codazzi equations),

ii) If $F(x, t)$ is the position vector in $\mathbb{R}^{n+1}$, then

$$
\Delta F(x, t)=-H(x, t) \nu(x, t) .
$$

Lemma 1.2 (Simons' Identity).

i) $\Delta h_{i j}=\nabla_{i} \nabla_{j} H+H h_{i l} h_{j}^{l}-|A|^{2} h_{i j}$,

ii) $2 h_{k}{ }^{j} \Delta h^{k}{ }_{j}=\Delta|A|^{2}-2|\nabla A|^{2}$,

iii) $\Delta|A|^{2}=2 h^{i j} \nabla_{i} \nabla_{j} H+2|\nabla A|^{2}+2 Z$.

The following lemmas are used in [Hu1] and [Hu2].

LEMMA 1.3. The trace-free part of the second fundamental form, $A^{0}=\left\{h_{i j}^{0}\right\}$ where $h_{i j}^{0}=h_{i j}-\frac{1}{n} H g_{i j}$, has the following properties:

i) $g^{i j} h_{i j}^{0}=0$ (hence the term trace-free),

ii) $\left|A^{0}\right|^{2}=|A|^{2}-\frac{1}{n} H^{2}$,

iii) $\left|\nabla A^{0}\right|^{2}=|\nabla A|^{2}-\frac{1}{n}|\nabla H|^{2}$

iv) $\left\langle\nabla_{i} H, \nabla_{i}\left(|A|^{2}-\frac{1}{n} H^{2}\right)\right\rangle=2\left\langle h_{k l}^{0} \nabla_{i} H, \nabla_{i} h_{k l}^{0}\right\rangle$.

\section{LEMMA 1.4.}

i) $|A|^{2} \geq \frac{1}{n} H^{2}$

ii) For weakly convex $M,|A|^{2} \leq H^{2}$.

LEMMA 1.5.

i) $\frac{1}{n}|\nabla H|^{2} \leq|\nabla A|^{2}$,

ii) $\left|\left\langle\nabla_{i}|A|^{2}, \nabla_{i} H\right\rangle\right| \leq 2 n H|\nabla A|^{2}$.

LEMMA 1.6.

i) $|\nabla A|^{2} \geq \frac{3}{n+2}|\nabla H|^{2}$

ii) Equivalently, $|\nabla A|^{2}-\frac{1}{n}|\nabla H|^{2} \geq \frac{2(n-1)}{3 n}|\nabla A|^{2}$.

LEMMA 1.7. Let $A=\left(a_{i j}\right)$ be a symmetric $n \times n$ matrix with eigenvalues $\lambda_{1}, \ldots, \lambda_{n}$. If for some $\lambda \in \mathbb{R}$,

$$
\lambda_{i} \geq \lambda
$$

for each $i=1, \ldots, n$, then each diagonal element of $A$ satisfies

$$
a_{i i} \geq \lambda
$$

This can be proved using a straightforward diagonalisation argument.

LEMMA 1.8. If $H>0$ and $h_{i j} \geq \varepsilon H g_{i j}$ with some $\varepsilon>0$, then

i) $Z \geq n \varepsilon^{2} H^{2}\left(|A|^{2}-\frac{1}{n}|H|^{2}\right)$,

ii) $\left|H \nabla_{i} h_{k l}-h_{k l} \nabla_{i} H\right|^{2} \geq \frac{1}{2} \varepsilon^{2} H^{2}|\nabla H|^{2}$,

iii) $n C-H|A|^{2} \geq 2 n \varepsilon H\left(|A|^{2}-\frac{1}{n}|H|^{2}\right)$.

Finally we will also need the following Bochner formula, the proof of which is a standard computation interchanging covariant derivatives and using the Gauss equations. 
LEMMA 1.9.

$$
\begin{aligned}
& \Delta|\nabla H|^{2}=2\left\langle\nabla_{l} \Delta H, \nabla_{l} H\right\rangle+2\left|\nabla^{2} H\right|^{2}+2 H\left\langle h_{l n}, \nabla_{l} H \nabla_{n} H\right\rangle \\
&-2\left\langle h_{i n} \nabla_{l} H, h_{i l} \nabla_{n} H\right\rangle .
\end{aligned}
$$

\section{Short time existence, preservation of convexity and elementary properties of the flow.}

Theorem 2.1 (Pihan, $[\mathrm{P}]$ ). For $\alpha \in(0,1)$, let $M_{0}$ be a $C^{3, \alpha}$ compact, embedded, $n$-dimensional submanifold in $\mathbb{R}^{n+1}$ without boundary, parametrised by a $C^{3, \alpha}$ embed$\operatorname{ding} F_{0}: \mathbb{R}^{n} \supset U \rightarrow F_{0}(U) \subset M_{0} \subset \mathbb{R}^{n+1}$. Assume also that $H \neq 0$ at some point of $M_{0}$. Then there exists $T_{0}>0$ such that the initial value problem

$$
\begin{gathered}
\frac{\partial}{\partial t} F(x, t)=\{1-h(t) H(x, t)\} \nu(x, t) \text { for } x \in U, t \in(0, T) \\
F(\cdot, 0) \equiv F_{0} \\
h(0)>0
\end{gathered}
$$

where $h(t)$ is given by (2), has a unique solution $F$ in $C^{3, \alpha}(U) \times C^{1, \frac{\alpha}{2}}\left(\left[0, T_{0}\right)\right)$.

In our case, $M_{0}$ is strictly convex, so clearly $H>0$ everywhere on $M_{0}$ and $h(0)>0$. As in $[\mathrm{P}]$, Theorem 2.1 implies short time continuity and positivity of various geometric quantities. As two important examples,

Corollary 2.2. $H>0$ for a short time.

COROLlaRY 2.3. $h(t)>0$ for a short time.

The 'short times' of Corollaries 2.2 and 2.3 may not be the same. When we refer to a solution on $[0, T)$, we always take the smallest $T>0$ necessary such that all relevant quantities are positive and finite.

A trivial modification to the proof of Hamilton's maximum principle for tensors on manifolds from [Ha] incorporates $h(t)>0$ in front of the Laplacian. Then, similarly as in [Hu1] and [Hu2], we have:

THEOREM 2.4. If $h_{i j} \geq 0$ at $t=0$, then it remains so on $[0, T)$.

THEOREM 2.5. If initially $H>0$ and $h_{i j} \geq \varepsilon H g_{i j}$ for some $\varepsilon \in\left[0, \frac{1}{n}\right)$, then $h_{i j} \geq \varepsilon H g_{i j}$ remains true on $[0, T)$.

We verify as in $[\mathrm{P}]$ that the surface area $\left|M_{t}\right|:=\int_{M_{t}} d \mu_{t}$ of the evolving hypersurface does indeed remain fixed under the flow (1), while the enclose volume does not decrease. For convenience, we parametrise $M_{t}$ over $\mathbb{S}^{n}$, which is possible since $M_{t}$ remains strictly convex for a short time by Corollary 2.2 and Theorem 2.5.

LEMMA 2.6. The surface area of $M_{t}$ remains constant throughout the flow, that is,

$$
\frac{d}{d t} \int_{M_{t}} d \mu_{t} \equiv 0
$$

Proof. We use the First Variation of Area Formula with the vector field $\frac{\partial F}{\partial t}$, extended appropriately, and the Divergence Theorem.

$$
\frac{d}{d t} \int_{M_{t}} d \mu_{t}=\int_{M_{t}} \operatorname{div}_{M_{t}}\left(\frac{\partial F}{\partial t}\right) d \mu_{t}=-\int_{M_{t}}(1-h H) H d \mu_{t} \equiv 0
$$


using (1) and (2).

LEMMA 2.7. The volume enclosed by $M_{t}$ does not decrease throughout the flow. That is, if $E_{t} \subset \mathbb{R}^{n+1}$ is the $(n+1)$-dimensional set enclosed by $M_{t}$, then

$$
\frac{d}{d t} \operatorname{Vol}\left(E_{t}\right) \geq 0 \text {. }
$$

Proof. Extend $F_{t}$ smoothly off $\mathbb{S}^{n}$ into all of $\mathbb{R}^{n+1}$ such that $F_{t}\left(B_{1}(O)\right)=E_{t}$ and $F_{t}\left(\mathbb{S}^{n}\right)=M_{t}=\partial E_{t}$. Then by the First Variation Formula,

$$
\begin{aligned}
\frac{d}{d t} \operatorname{Vol}\left(E_{t}\right)=\int_{E_{t}} \operatorname{div}_{\mathbb{R}^{n+1}}\left(\frac{\partial F}{\partial t}\right) d V & =\int_{M_{t}}\left\langle\frac{\partial F}{\partial t}, \nu\right\rangle d \mu_{t} \\
& =\int_{M_{t}}(1-h H) d \mu_{t}=\int_{M_{t}} d \mu_{t}-\frac{\left(\int_{M_{t}} H d \mu_{t}\right)^{2}}{\left(\int_{M_{t}} H^{2} d \mu_{t}\right)} \geq 0
\end{aligned}
$$

using (1), (2) and the Cauchy-Schwarz inequality.

REMARK. If $H$ is constant, then we have equality in Lemma 2.7. Hence if $M_{0}$ is a sphere, then the volume enclosed by $M_{t}$ remains constant under the flow. This is consistent with the observation from equation (2) that if $H$ is constant then $h \equiv \frac{1}{H}$ and (1) becomes $\frac{\partial F}{\partial t} \equiv 0$. Thus a sphere remains static under this flow.

We now state our main theorem to be proved.

THEOREM 2.8. If the initial $n$-dimensional hypersurface $M_{0}, n \geq 2$, is strictly convex, then the evolution equation (1) has a smooth solution $M_{t}$ for all times $0 \leq$ $t<\infty$, and the $M_{t}$ 's converge, as $t \rightarrow \infty$, in the $C^{\infty}$-topology, to a sphere with the same surface area as $M_{0}$.

3. The solution remains within a bounded region of $\mathbb{R}^{n+1}$. In this section we show that the solution hypersurface $M_{t}$ remains within a large ball whose radius depends only on the initial hypersurface $M_{0}$ and is, in particular, independent of time. The result is based upon a theorem from [CG].

\section{Notation}

- $\nabla$ and $g$ denote the covariant derivative and metric on $\mathbb{S}^{n}$.

- $\mathbb{R}_{+}=\{a \in \mathbb{R}: a>0\}$.

- For any unit vector $\eta \in \mathbb{S}^{n} \subset \mathbb{R}^{n+1}$,

$$
\mathbb{S}_{+}^{n}=\mathbb{S}_{+}^{n}(\eta)=\left\{x \in \mathbb{S}^{n}:\langle x, \eta\rangle \geq 0\right\} .
$$

Theorem 3.1 (Chow, Gulliver, $[\mathrm{CG}])$. Let $u \in C^{2}\left(\mathbb{S}^{n} \times[0, T)\right), 0<T \leq \infty$, be a solution to the PDE

$$
\begin{gathered}
u_{t}=G\left(\nabla^{2} u+g u, t\right) \text { for all }(x, t) \in \mathbb{S}^{n} \times(0, T) \\
\qquad u(\cdot, 0) \equiv u_{0}
\end{gathered}
$$

where the following hold: 
a) $G$ is invariant under similarity transformations of the matrix $\nabla^{2} u+g u$. $G$ depends only on the eigenvalues $r_{1}, \ldots, r_{n}$ of $\nabla^{2} u+g u$.

b) $G$ is differentiable with respect to each $r_{i}$ and $\frac{\partial G}{\partial r_{i}} \geq 0$ for each $i$. Then

c) $u_{0}$ is Lipschitz.

i) Given any unit vector $\eta \in \mathbb{S}^{n}$, there is a $\lambda=\lambda\left(u_{0}\right) \in \mathbb{R}_{+}$such that, for all $(x, t) \in \mathbb{S}_{+}^{n} \times[0, T)$

$$
u(x-2\langle x, \eta\rangle \eta, t)+\lambda\langle x, \eta\rangle \geq u(x, t) .
$$

ii) With the same $\lambda$ as in i), for all $x_{1}, x_{2} \in \mathbb{S}^{n}, t \in[0, T)$,

$$
\left|u\left(x_{1}, t\right)-u\left(x_{2}, t\right)\right| \leq \lambda\left|\sin \left(\frac{1}{2} \operatorname{dist}_{\mathbb{S}^{n}}\left(x_{1}, x_{2}\right)\right)\right| .
$$

iii) $\max _{x \in \mathbb{S}^{n}} u(x, t)-\min _{x \in \mathbb{S}^{n}} u(x, t) \leq \lambda$ for all $t \in[0, T)$.

iv) $|\nabla u(x, t)| \leq \frac{\lambda}{2}$ for all $(x, t) \in \mathbb{S}^{n} \times[0, T)$.

REMARK. Chow and Gulliver consider $G$ which depends on the slightly more general matrix $\nabla^{2} u+c g u$, where $c \leq 1$ is a constant. Their $G$ also depends explicitly upon $u$. Consequently they require $G$ to satisfy a monotonicity condition.

Unlike Chow and Gulliver, we have stated Theorem 3.1 without a Lipschitz continuity hypothesis on $G$. Then the theorem can be applied to the $G$ 's arising for many curvature flows including the surface area preserving mean curvature flow. The proof remains the same, except that the comparison principle for nonlinear parabolic PDEs is used instead of the Lipschitz continuity of $G$.

We will apply Theorem 3.1 to the real-valued support function of $M_{t}$, defined as in [An1] and [U]. An alternative, equivalent definition, along with elementary properties of the support function, can be found in $[\mathrm{L}]$.

Definition. The support function $Z: \mathbb{S}^{n} \rightarrow \mathbb{R}$ of a strictly convex hypersurface $M=F\left(\mathbb{S}^{n}\right)$ is given by

$$
Z(x)=\left\langle F\left(\nu^{-1}(x)\right), x\right\rangle
$$

where $\nu^{-1}: \mathbb{S}^{n} \rightarrow \mathbb{S}^{n}$ is the inverse Gauss map of $M$.

Geometrically, the support function $Z(x)$ gives the perpendicular distance to the origin of the tangent plane $T_{F\left(\nu^{-1}(x)\right)} M$. [An1].

$M$ may be parametrised using its support function. The following result is from

LEMMA 3.2. If $M$ is a strictly convex, compact hypersurface with support function $Z: \mathbb{S}^{n} \rightarrow \mathbb{R}$ then $M$ can be parametrised such that its Gauss map is the identity, by the immersion $\bar{F}: \mathbb{S}^{n} \rightarrow \mathbb{R}^{n+1}$ where

$$
\bar{F}(x)=Z(x) x+\nabla Z(x) .
$$

As the flow (1) preserves strict convexity for a short time, the support function of $M_{t}, Z(\cdot, t): \mathbb{S}^{n} \rightarrow \mathbb{R}$, can be defined by

$$
Z(x, t)=\left\langle F\left(\nu_{t}^{-1}(x), t\right), x\right\rangle
$$


where $\nu_{t}^{-1}: \mathbb{S}^{n} \rightarrow \mathbb{S}^{n}$ is the inverse Gauss map of $M_{t}$.

It is straightforward to derive, similarly as in [U], the evolution equation for the support function under the flow (1).

LEMMA 3.3. Under the flow (1),

$$
\frac{\partial Z}{\partial t}(x, t)=1-h(t) H\left(\nu_{t}^{-1}(x), t\right)
$$

REMARK. The parametrisation in Lemma 3.2 will not be preserved by the flow unless we incorporate a tangential diffeomorphism. However, here we will only need to use Lemma 3.2 at a fixed time.

Also as in [U] we note that the support function of $M_{t}$ and the inverse of the Weingarten map of $M_{t}, \mathcal{W}^{-1}$ are related by

$$
\mathcal{W}^{-1}\left(\nu_{t}^{-1}(x), t\right)=\nabla^{2} Z(x, t)+Z(x, t) g .
$$

Hence the mean curvature is given in terms of the support function by

$$
H\left(\nu_{t}^{-1}(x), t\right)=\operatorname{trace}\left\{\left(\nabla^{2} Z(x, t)+Z(x, t) g\right)^{-1}\right\} \text {. }
$$

Proposition 3.4. For $t \in[0, T), M_{t} \subset B_{r}(O)$, where $r=r\left(M_{0}\right)$.

Proof. Fix $t \in[0, T)$ and set $P=\max _{x \in \mathbb{S}^{n}} Z\left(x, t_{0}\right)$. Comparing equation (5) with (4), in view of (6) and (7),

$$
G=1-h(t) \sum_{i=1}^{n} \frac{1}{r_{i}},
$$

and so

$$
\frac{\partial G}{\partial r_{i}}=h(t) \cdot \frac{1}{r_{i}^{2}} \geq 0
$$

by Corollary 2.3. By Theorem 2.5 and Corollary 2.2, strict convexity is preserved for $t \in[0, T)$. Hence Theorem 3.1 can be applied to (5); part iii) gives that there is a $\lambda=\lambda\left(M_{0}\right)$ such that

$$
\min _{x \in \mathbb{S}^{n}} Z\left(x, t_{0}\right) \geq P-\lambda .
$$

Hence, assuming $P>\lambda, B_{P-\lambda}(O) \subset E_{t}$. By Corollary 2.2 and Theorem $2.5, M_{t}$ is strictly convex, so

$$
\left|M_{t}\right| \geq\left|B_{P-\lambda}(O)\right|
$$

Lemma 2.6 therefore implies

$$
\left|M_{0}\right| \geq(n+1) \omega_{n+1}(P-\lambda)^{n}
$$

and hence

$$
P \leq \lambda+\left(\frac{\left|M_{0}\right|}{(n+1) \omega_{n+1}}\right)^{\frac{1}{n}} .
$$


Finally, combining this with by Lemma 3.2,

$$
\left|F\left(x, t_{0}\right)\right| \leq\left|Z\left(x, t_{0}\right)\right|+\left|\nabla Z\left(x, t_{0}\right)\right| \leq \frac{3 \lambda}{2}+\left(\frac{\left|M_{0}\right|}{(n+1) \omega_{n+1}}\right)^{\frac{1}{n}},
$$

where we have also used Theorem 3.1, iv).

Hence the result, with $r=\frac{3 \lambda}{2}+\left(\frac{\left|M_{0}\right|}{(n+1) \omega_{n+1}}\right)^{\frac{1}{n}}$.

REMARKS.

1. As consequences of this result, the diameter of $M_{t}$ remains bounded under the flow and $M_{t}$ does not drift out to $\infty$ in some direction.

2. A analogous argument to the above can be used to show solutions of the volume preserving mean curvature flow, considered in [Hu2], remain within a suitably large ball, using the fixed volume instead of the fixed area. The argument would also work for other constrained flows such as those in [M].

3. Using Chow and Gulliver's theorem we have achieved this result before obtaining uniform bounds on curvatures and their derivatives, in contrast to the method of Huisken in [Hu2].

4. Evolution equations. The following evolution equations for the surface are preserving mean curvature flow may be derived similarly as in [Hu1] or [P]. From now on, $\nabla$ and $\Delta$ denote the covariant derivative and Laplace-Beltrami operator on $M_{t}$.

LEMMA 4.1. The metric of the evolving hypersurface $M_{t}$ satisfies

$$
\frac{\partial}{\partial t} g_{i j}=2(1-h H) h_{i j}
$$

COROLlary 4.2 .

i) $\frac{\partial}{\partial t} g^{i j}=-2(1-h H) h^{i j}$,

ii) $\frac{\partial}{\partial t} \mu_{t}=H(1-h H) \mu_{t}$ where $\mu_{t}=\sqrt{\operatorname{det}\left(g_{i j}\right)}$.

LEMMA 4.3. The outward unit normal to $M_{t}$ evolves according to

$$
\frac{\partial}{\partial t} \nu=h \nabla H
$$

THEOREM 4.4. The second fundamental form of $M_{t}$ evolves by

$$
\frac{\partial}{\partial t} h_{i j}=h \Delta h_{i j}+(1-2 h H) h_{i}^{m} h_{m j}+h|A|^{2} h_{i j} .
$$

Corollary 4.5.
i) $\frac{\partial}{\partial t} h^{i}{ }_{j}=h\left(\Delta h^{i}{ }_{j}+|A|^{2} h^{i}{ }_{j}\right)-h_{m}^{i} h^{m}{ }_{j}$,
ii) $\frac{\partial}{\partial t} H=h \Delta H-(1-h H)|A|^{2}$,
iii) $\frac{\partial}{\partial t}|A|^{2}=h\left(\Delta|A|^{2}-2|\nabla A|^{2}+2|A|^{4}\right)-2 C$.

COROLlary 4.6.

i) $\frac{\partial}{\partial t} H^{2}=h \Delta H^{2}-2 h|\nabla H|^{2}-2(1-h H) H|A|^{2}$,

ii) $\frac{\partial}{\partial t}\left(|A|^{2}-\frac{1}{n} H^{2}\right)=h \Delta\left(|A|^{2}-\frac{1}{n} H^{2}\right)-2 h\left(|\nabla A|^{2}-\frac{1}{n}|\nabla H|^{2}\right)$

$$
+2 h|A|^{2}\left(|A|^{2}-\frac{1}{n} H^{2}\right)-2 C+\frac{2}{n} H|A|^{2} \text {. }
$$


5. Positive bounds on $h(t)$. By Proposition 3.4, the diameter of $M_{t}$ is bounded by $d:=2 r\left(M_{0}\right)$ under the flow. We will use the diameter bound in this section, however, we remark that in fact, upper and lower positive bounds on $h(t)$ can be obtained without Proposition 3.4, see Appendix A.

LEMMA 5.1. For $t \in[0, T), \int_{M_{t}} H d \mu_{t} \geq \frac{n\left|M_{0}\right|}{d}$.

Proof. Applying the Divergence Theorem to the position vector $F$ yields

$$
n\left|M_{0}\right|=n\left|M_{t}\right| \leq \int_{M_{t}} H|\langle F, \nu\rangle| d \mu_{t} \leq \int_{M_{t}} H|F| d \mu_{t} \leq d \int_{M_{t}} H d \mu_{t}
$$

using Lemmas 2.6 and the diameter bound. Hence the result.

Applying the Cauchy-Schwarz inequality to Lemma 5.1, we immediately obtain:

Corollary 5.2. For $t \in[0, T), \int_{M_{t}} H^{2} d \mu_{t} \geq \frac{n^{2}\left|M_{0}\right|}{d^{2}}$.

REMARK. For $n=2$ we could alternatively use the elementary bound from [W],

$$
\int_{M_{t}} H^{2} d \mu_{t} \geq 4 \pi
$$

This is obtained as follows. Contracting the Gauss equations, (3), twice yields

$$
R=H^{2}-|A|^{2}
$$

where $R$ is the scalar curvature. Therefore

$$
H^{2}=|A|^{2}+R \geq R
$$

For $n=2$,

$$
R=2 K
$$

where $K$ is the Gauss curvature of $M_{t}$. The Gauss-Bonnet Theorem gives

$$
\int_{M_{t}} K d \mu_{t}=2 \pi
$$

since the surfaces $M_{t}$ have genus equal to one. Integrating (10) over $M_{t}$ and using (11) and (12) on the right hand side yields (8).

Using the Cauchy-Schwarz inequality, Lemma 2.6 and Corollary 5.2, we easily obtain:

Corollary 5.3. For $t \in[0, T), h(t) \leq \frac{d}{n}:=S\left(n, M_{0}\right)$.

For an upper bound on $\int_{M_{t}} H^{2} d \mu_{t}$ we apply the maximum principle to the evolution equation for $\frac{|A|^{2}}{H^{2}}$ and also use an Aleksandrov-Fenchel type inequality for mixed volumes. The quantity $\frac{|A|^{2}}{H^{2}}$ was considered by Huisken in [Hul] and [Hu3].

LEMMA 5.4. For $t \in[0, T)$,

$$
\frac{\partial}{\partial t}\left(\frac{|A|^{2}}{H^{2}}\right) \leq h \Delta\left(\frac{|A|^{2}}{H^{2}}\right)+\frac{h}{H^{2}}\left\langle\nabla_{i}\left(\frac{|A|^{2}}{H^{2}}\right), \nabla_{i} H^{2}\right\rangle .
$$


REMARK. Huisken derived a very similar inequality for the original mean curvature flow in [Hu3], namely

$$
\frac{\partial}{\partial t}\left(\frac{|A|^{2}}{H^{2}}\right) \leq \Delta\left(\frac{|A|^{2}}{H^{2}}\right)+\frac{1}{H^{2}}\left\langle\nabla_{i}\left(\frac{|A|^{2}}{H^{2}}\right), \nabla_{i} H^{2}\right\rangle
$$

It is remarkable that such a similar inequality holds for the significantly different surface area preserving mean curvature flow. The inequality has the ideal form for applying the weak maximum principle!

Proof of Lemma. Using Corollaries 4.5, iii) and 4.6, i), we compute

$$
\begin{aligned}
\frac{\partial}{\partial t}\left(\frac{|A|^{2}}{H^{2}}\right)=h \Delta\left(\frac{|A|^{2}}{H^{2}}\right) & +\frac{2 h}{H^{2}}\left\langle\nabla_{i}\left(\frac{|A|^{2}}{H^{2}}\right), \nabla_{i} H^{2}\right\rangle \\
& -2 h\left(\frac{H^{2}|\nabla A|^{2}-|A|^{2}|\nabla H|^{2}}{H^{4}}\right)-\frac{2}{H^{3}}\left(H C-|A|^{4}\right) .
\end{aligned}
$$

Noting that

$$
H^{2}|\nabla A|^{2}-|A|^{2}|\nabla H|^{2}=\left|H \nabla_{i} h_{j k}-h_{j k} \nabla_{i} H\right|^{2}+H^{3}\left\langle\nabla_{i}\left(\frac{|A|^{2}}{H^{2}}\right), \nabla_{i} H\right\rangle,
$$

(13) becomes

$$
\begin{aligned}
& \frac{\partial}{\partial t}\left(\frac{|A|^{2}}{H^{2}}\right)=h \Delta\left(\frac{|A|^{2}}{H^{2}}\right)+\frac{2 h}{H^{2}}\left\langle\nabla_{i}\left(\frac{|A|^{2}}{H^{2}}\right), \nabla_{i} H^{2}\right\rangle-\frac{2}{H^{3}}\left(H C-|A|^{4}\right) \\
&-\frac{2 h}{H^{4}}\left(\left|H \nabla_{i} h_{j k}-h_{j k} \nabla_{i} H\right|^{2}+H^{3}\left\langle\nabla_{i}\left(\frac{|A|^{2}}{H^{2}}\right), \nabla_{i} H\right\rangle\right) \\
& \leq h \Delta\left(\frac{|A|^{2}}{H^{2}}\right)+\frac{h}{H^{2}}\left\langle\nabla_{i}\left(\frac{|A|^{2}}{H^{2}}\right), \nabla_{i} H^{2}\right\rangle
\end{aligned}
$$

using Lemma 1.8, i) with Lemma 1.4, i) and Corollary 2.2 .

Corollary 5.5. There is a $\delta \in(0,1)$, depending only on $M_{0}$, such that, for $t \in[0, T)$,

$$
|A|^{2}(x, t) \leq(1-\delta) H^{2}(x, t)
$$

Proof. In view of Lemma 5.4, the maximum principle is applicable to $\frac{|A|^{2}}{H^{2}}$. This gives

$$
\frac{|A|^{2}}{H^{2}}(\cdot, t) \leq \max _{M_{0}}\left(\frac{|A|^{2}}{H^{2}}\right)=C_{0}=C_{0}\left(M_{0}\right)
$$

Now the constant $C_{0} \leq 1$ by Lemma 1.4 , ii) and Theorem 2.4. In fact, $C_{0}<1$, since if $\max _{M_{0}}\left(\frac{|A|^{2}}{H^{2}}\right)=1$, then at some point $p$ of $M_{0}$, by compactness,

$$
|A|^{2}=H^{2} \text {. }
$$


In terms of the principal curvatures of $M_{0}$, this would mean that

$$
\sum_{i<j} \kappa_{i} \kappa_{j}=0
$$

at $p$, which is not possible for a strictly convex initial hypersurface $M_{0}$. Hence from (14), for some $\delta \in(0,1)$, for each $x \in \mathbb{S}^{n}$ and all $t \in[0, T)$,

$$
|A|^{2}(x, t) \leq C_{0} H^{2}(x, t)=(1-\delta) H^{2}(x, t) .
$$

$\square$

Corollary 5.6. For $t \in[0, T)$,

$$
\int_{M_{t}} H^{2} d \mu_{t} \leq C=C\left(n, M_{0}\right)
$$

Proof. Consider the case of $n=2$ first. Using (9), (11) and the Gauss-Bonnet Theorem, (12),

$$
\pi=\int_{M_{t}}\left(H^{2}-|A|^{2}\right) d \mu_{t} \geq \delta \int_{M_{t}} H^{2} d \mu_{t}
$$

using also Corollary 5.5. The result follows.

For $n>2$, the Gauss-Bonnet theorem cannot so easily be applied. However, the Aleksandrov-Fenchel inequality for mixed volumes can be employed. ${ }^{1}$ If we write

$$
\int_{M_{t}} H^{2} d \mu_{t}=\int_{M_{t}}|A|^{2} d \mu_{t}+2 \int_{M_{t}} \sum_{i<j} \kappa_{i} \kappa_{j} d \mu_{t},
$$

the second term on the right of (16) is a mixed volume. As shown in Appendix A,

$$
\int_{M_{t}} \sum_{i<j} \kappa_{i} \kappa_{j} d \mu_{t} \leq U\left(n, M_{0}\right)
$$

for some constant $U$. Using this in (16) together with Corollary 5.5 gives

$$
\int_{M_{t}} H^{2} d \mu_{t} \leq(1-\delta) \int_{M_{t}} H^{2} d \mu_{t}+U
$$

and hence the result.

Combining Corollary 5.6 and Lemma 5.1 we get:

Corollary 5.7. For $t \in[0, T), h(t) \geq L=L\left(n, M_{0}\right)>0$.

6. A pinching estimate. As in [Hu1] and [Hu2], the quantity

$$
\frac{1}{n} \sum_{i<j}^{n}\left(\kappa_{i}-\kappa_{j}\right)^{2}=|A|^{2}-\frac{1}{n} H^{2}
$$

a measure of the difference between the principal curvatures, is bounded by a power of the mean curvature.

\footnotetext{
${ }^{1}$ I would like to thank Dr Ben Andrews for suggesting this.
} 
Theorem 6.1. There exists $a \delta>0$ and $a C_{0}<\infty$, depending only on $n$ and $M_{0}$ such that, for $t \in[0, T)$,

$$
|A|^{2}-\frac{1}{n} H^{2} \leq C_{0} H^{2-\delta}
$$

Let $f_{\sigma}=\frac{1}{H^{\alpha}}\left(|A|^{2}-\frac{1}{n} H^{2}\right), \alpha=2-\sigma, \sigma \in(0,2)$. Theorem 6.1 will be proven by bounding $f_{\sigma}$. From [Hu1] and [Hu2], we have:

LEMMA 6.2.

i) $\frac{2}{H^{\alpha}}\left(|\nabla A|^{2}-\frac{1}{n}|\nabla H|^{2}\right)=\frac{2}{H}\left\langle\nabla_{i} f_{\sigma}, \nabla_{i} H\right\rangle+\frac{2(\alpha-1)}{H^{2}} f_{\sigma}|\nabla H|^{2}$

ii) $\frac{2}{n H^{\alpha}}\left(H|A|^{2}-n C\right)+\frac{\alpha|A|^{2}}{H} f_{\sigma}=\frac{2}{H^{\alpha+1}}\left(|A|^{4}-H C\right)-\sigma|A|^{2} \frac{f_{\sigma}}{H}$,

$$
+\frac{2}{H^{\alpha+2}}\left|H \nabla_{i} h_{k l}-h_{k l} \nabla_{i} H\right|^{2}
$$

iii) $\Delta f_{\sigma}=\frac{2}{H^{\alpha}}\left\langle h_{i j}^{0}, \nabla_{i} \nabla_{j} H\right\rangle+\frac{2}{H^{\alpha}} Z+\frac{2}{H^{\alpha+2}}\left|H \nabla_{i} h_{k l}-h_{k l} \nabla_{i} H\right|^{2}$

iv) Let $p \geq 2$. Then for any $\eta>0$ and any $0 \leq \sigma \leq \frac{1}{2}$,

$$
-\frac{\alpha}{H} f_{\sigma} \Delta H+\frac{(2-\alpha)(\alpha-1)}{H^{2}} f_{\sigma}|\nabla H|^{2}-\frac{2(\alpha-1)}{H}\left\langle\nabla_{i} H, \nabla_{i} f_{\sigma}\right\rangle \text {, }
$$

$n \varepsilon^{2} \int_{M_{t}} f_{\sigma}^{p} H^{2} d \mu_{t} \leq(2 \eta p+5) \int_{M_{t}} \frac{1}{H^{\alpha}} f_{\sigma}^{p-1}|\nabla H|^{2} d \mu_{t}$

Using Corollary 4.5, ii),

$$
+\frac{1}{\eta}(p-1) \int_{M_{t}} f_{\sigma}^{p-2}\left|\nabla f_{\sigma}\right|^{2} d \mu_{t} .
$$

Lemma 6.3. For $t \in[0, T)$,

$$
\frac{\partial}{\partial t} H^{\alpha}=h \Delta H^{\alpha}-\alpha(\alpha-1) h H^{\alpha-2}|\nabla H|^{2}-\alpha(1-h H) H^{\alpha-1}|A|^{2} .
$$

Together with Corollary 4.6, we obtain

Lemma 6.4. For $t \in[0, T)$,

$$
\begin{aligned}
\frac{\partial}{\partial t} f_{\sigma}= & h \Delta f_{\sigma}+\frac{2 h(\alpha-1)}{H}\left\langle\nabla_{i} f_{\sigma}, \nabla_{i} H\right\rangle-\frac{2 h}{H^{\alpha+2}}\left|H \nabla_{i} h_{k l}-h_{k l} \nabla_{i} H\right|^{2} \\
& -\frac{h}{H^{2}}(\alpha-1)(2-\alpha) f_{\sigma}|\nabla H|^{2}+\frac{2}{H^{\alpha+1}}\left(|A|^{4}-H C\right) \\
& -\sigma \frac{|A|^{2}}{H}(1-h H) .
\end{aligned}
$$

In view of Corollary 2.2 and Theorem 2.5, the inequalities of Lemma 1.8, i) and ii) hold, so from Lemma 6.4 , if we assume $\sigma \in(0,1)$, then

Corollary 6.5. For $t \in[0, T)$,

$$
\begin{aligned}
\frac{\partial}{\partial t} f_{\sigma} \leq h\left\{\Delta f_{\sigma}+\frac{2(\alpha-1)}{H}\left\langle\nabla_{i} f_{\sigma}, \nabla_{i} H\right\rangle-\frac{\varepsilon^{2}}{H^{\alpha}}|\nabla H|^{2}\right. & \left.+\sigma|A|^{2} f_{\sigma}\right\} \\
& -2 \varepsilon^{2} H f_{\sigma}-\sigma \frac{|A|^{2}}{H} f_{\sigma} .
\end{aligned}
$$

This inequality is very similar to those obtained in [Hu1] and [Hu2]. Consequently, the following analysis proceeds similarly. However here we must also use that $h(t)>0$ by Corollary 2.3 .

LEMMA 6.6. For all $p \geq \max \left(2,100 \varepsilon^{-2}\right)$ and $\sigma \leq \min \left(\frac{1}{2}, \frac{n}{8} \varepsilon^{3} p^{-\frac{1}{2}}\right)$, we have for all $t \in[0, T)$,

$$
\left(\int_{M_{t}} f_{\sigma}^{p} d \mu_{t}\right)^{\frac{1}{p}} \leq c_{1}=c_{1}\left(M_{0}\right)<\infty
$$


Proof. Choose

$$
c_{1}=\left(\left|M_{0}\right|+1\right) \sup _{\sigma \in\left[0, \frac{1}{2}\right]} \sup _{M_{0}} f_{\sigma}
$$

It then suffices to show that for $t \in[0, T)$,

$$
\frac{d}{d t} \int_{M_{t}} f_{\sigma}^{p} d \mu_{t} \leq 0
$$

Multiplying (17) by $p f_{\sigma}^{p-1}$ and integrating over $M_{t}$, we derive similarly as in [Hu1] and [Hu2], using Corollary 4.2 and the assumption on $p$,

$$
\begin{aligned}
\frac{d}{d t} \int_{M_{t}} f_{\sigma}^{p} d \mu_{t}+\frac{h}{2} p(p & -1) \int_{M_{t}} f_{\sigma}^{p-2}\left|\nabla f_{\sigma}\right|^{2} d \mu_{t}+\frac{1}{2} \varepsilon^{2} h p \int_{M_{t}} \frac{1}{H^{\alpha}} f_{\sigma}^{p-1}|\nabla H|^{2} d \mu_{t} \\
& \leq\left(1-2 p \varepsilon^{2}-\sigma p\right) \int_{M_{t}} f_{\sigma}^{p} H d \mu_{t}+(\sigma p-1) h \int_{M_{t}} f_{\sigma}^{p} H^{2} d \mu_{t} .
\end{aligned}
$$

Therefore, using Corollary 2.3,

$$
\begin{aligned}
\frac{d}{d t} \int_{M_{t}} f_{\sigma}^{p} d \mu_{t}+\frac{h}{2} p(p-1) \int_{M_{t}} f_{\sigma}^{p-2}\left|\nabla f_{\sigma}\right|^{2} d \mu_{t}+\frac{1}{2} \varepsilon^{2} h p \int_{M_{t}} \frac{1}{H^{\alpha}} f_{\sigma}^{p-1}|\nabla H|^{2} d \mu_{t} \\
\quad \leq \sigma p h \int_{M_{t}} f_{\sigma}^{p} H^{2} d \mu_{t} \\
\leq h \frac{n}{8} \varepsilon^{3} p^{\frac{1}{2}} \int_{M_{t}} f_{\sigma}^{p} H^{2} d \mu_{t} \text { by assumption on } \sigma \\
\leq h \frac{\varepsilon}{8} p^{\frac{1}{2}}\left\{(2 \eta p+5) \int_{M_{t}} \frac{1}{H^{\alpha}} f_{\sigma}^{p-1}|\nabla H|^{2} d \mu_{t}+\frac{(p-1)}{\eta} \int_{M_{t}} f_{\sigma}^{p-2}\left|\nabla f_{\sigma}\right|^{2} d \mu_{t}\right\} \\
\leq h\left(\frac{\varepsilon^{2}}{16} p+\frac{5 \varepsilon}{8} p^{\frac{1}{2}}\right) \int_{M_{t}} \frac{1}{H^{\alpha}} f_{\sigma}^{p-1}|\nabla H|^{2} d \mu_{t}+\frac{h p(p-1)}{2} \int_{M_{t}} f_{\sigma}^{p-2}\left|\nabla f_{\sigma}\right|^{2} d \mu_{t}
\end{aligned}
$$

where we have used Lemma 6.2 , iv) with $\eta=\frac{\varepsilon}{4} p^{-\frac{1}{2}}$. Therefore

$$
\frac{d}{d t} \int_{M_{t}} f_{\sigma}^{p} d \mu_{t} \leq h\left(\frac{5 \varepsilon}{8} p^{\frac{1}{2}}-\frac{7 \varepsilon^{2}}{16} p\right) \int_{M_{t}} \frac{1}{H^{\alpha}} f_{\sigma}^{p-1}|\nabla H|^{2} d \mu_{t}<0
$$

since $h(t)>0$ by Corollary 2.3 and $p^{\frac{1}{2}} \geq \frac{10}{\varepsilon}$. This completes the proof.

Exactly as in [Hul], we then have

Corollary 6.7. If $p \geq \max \left(2,100 \varepsilon^{-2},\left(\frac{m}{n}\right)^{2} 2^{8} \varepsilon^{-6}\right)$ and $\sigma \leq \min \left(\frac{1}{2}, \frac{n}{8} \varepsilon^{3} p^{-\frac{1}{2}}, \frac{n}{16} \varepsilon^{3} p^{-\frac{1}{2}}\right)$, then for $t \in[0, T)$,

$$
\left(\int_{M_{t}} H^{m} f_{\sigma}^{p} d \mu_{t}\right)^{\frac{1}{p}} \leq c_{1}
$$

To prove Theorem 6.1 we also need three well known results: 
Lemma 6.8 (Michael-Simon Sobolev inequality, [MS]). For all Lipschitz functions $v$ on $M$,

$$
\left(\int_{M}|v|^{\frac{n}{n-1}} d \mu_{t}\right)^{\frac{n-1}{n}} \leq c_{s}(n)\left(\int_{M}|\nabla v| d \mu_{t}+\int_{M}|H||v| d \mu_{t}\right) .
$$

Corollary 6.9. For all Lipschitz functions $v$ on $M$, if $A=\{x \in M: v \neq 0\}$ and $q=\frac{n}{n-1}$ then

$$
\begin{aligned}
\left(\int_{M} v^{2 q} d \mu\right)^{\frac{1}{q}} \leq c_{w}(n,|M|) \int_{M}|\nabla v|^{2} d \mu & \\
& +\tilde{c}_{s}(n)\left(\int_{A}|H|^{n} d \mu\right)^{\frac{2}{n}}\left(\int_{M} v^{2 q} d \mu\right)^{\frac{1}{q}}
\end{aligned}
$$

LEMMA 6.10. For $r \leq s \leq w \leq$ and $\frac{1}{s}=\frac{\theta}{r}+\frac{1-\theta}{w}$, if $u \in L^{r}(M) \cap L^{w}(M)$ then $u \in L^{s}(M)$ and

$$
\|u\|_{L^{s}(M)} \leq\|u\|_{L^{r}(M)}^{\theta}\|u\|_{L^{w}(M)}^{(1-\theta)}
$$

Lemma 6.11 (Stampacchia, $[\mathrm{St}])$. Let $\varphi:\left[k_{1}, \infty\right)$ be a nonnegative, nonincreasing function such that

$$
\varphi(h) \leq \frac{\tilde{C}}{(h-k)^{\tilde{p}}}|\varphi(k)|^{\gamma}
$$

for all $h>k \geq k_{1}$, where $\tilde{C}, \tilde{p}$ and $\gamma$ are positive constants and $\gamma>1$. Then

$$
\varphi\left(k_{1}+D\right)=0
$$

where $D^{\tilde{p}}=2^{\frac{\gamma \tilde{p}}{\gamma-1}} \tilde{C}\left|\varphi\left(k_{1}\right)\right|^{\gamma-1}$.

Proof of Theorem 6.1. Following [Hu1] and [Hu2], define pointwise on $M_{t}$

$$
f_{\sigma, k}=\max \left(f_{\sigma}-k, 0\right)
$$

for all $k \geq k_{0}=\sup _{M_{0}} f_{\sigma}$. For each $t, f_{\sigma, k}$ is nonzero on the set

$$
A(k)_{t}=\left\{x \in M_{t}: f_{\sigma}>k\right\} \text {. }
$$

This set will be denoted simply by $A$ or $A(k)$ where no confusion could arise.

Multiplying inequality (17) by $p f_{\sigma, k}^{p-1}$, we derive similarly as in [Hu1] and [Hu2],

$$
\frac{d}{d t} \int_{A} f_{\sigma, k}^{p} d \mu_{t}+\frac{h}{2} p(p-1) \int_{A} f_{\sigma, k}^{p-2}\left|\nabla f_{\sigma}\right|^{2} d \mu_{t} \leq h \sigma p \int_{A} f_{\sigma, k}^{p-1} f_{\sigma} H^{2} d \mu_{t}
$$

Almost everywhere on $A(k)$,

$$
\frac{1}{2} p(p-1) f_{\sigma, k}^{p-2}\left|\nabla f_{\sigma}\right|^{2} \geq\left|\nabla f_{\sigma, k}^{\frac{p}{2}}\right|^{2}
$$


so with $v=f_{\sigma, k}^{\frac{p}{2}}$ and using $f_{\sigma, k} \leq f_{\sigma}$ on $A(k)_{t}$,

$$
\frac{d}{d t} \int_{A} v^{2} d \mu_{t}+\int_{A}|\nabla v|^{2} d \mu_{t} \leq h \sigma p \int_{A} f_{\sigma}^{p} H^{2} d \mu_{t}
$$

Recalling Lemma 2.6, we now apply Corollary 6.9 to the $\nabla v$ term to obtain

$$
\left\{1-\tilde{c}_{s}(n)\left(\int_{A}|H|^{n} d \mu_{t}\right)^{\frac{2}{n}}\right\}\left(\int_{M_{t}} v^{2 q} d \mu_{t}\right)^{\frac{1}{q}} \leq c_{w}\left(n,\left|M_{0}\right|\right) \int_{M_{t}}|\nabla v|^{2} d \mu_{t} .
$$

Now,

$$
\left(\int_{A} H^{n} d \mu_{t}\right)^{\frac{2}{n}} \leq k^{-\frac{2 p}{n}}\left(\int_{A} H^{n} f_{\sigma}^{p} d \mu_{t}\right)^{\frac{2}{n}} \leq k^{-\frac{2 p}{n}} c_{1}^{\frac{2 p}{n}}
$$

by Corollary 6.7 with $m=n$, provided $p \geq 2^{8} \varepsilon^{-6}$ and $\sigma<\frac{n}{16} \varepsilon^{3} p^{-\frac{1}{2}}$. Choose $k=k_{1}$ large enough such that $1-\tilde{c}_{s}(n)\left(\int_{A}|H|^{n} d \mu_{t}\right)^{\frac{2}{n}} \geq \frac{1}{2}$. Then (21) becomes

$$
\left(\int_{M_{t}} v^{2 q} d \mu_{t}\right)^{\frac{1}{q}} \leq 2 c_{w}\left(n,\left|M_{0}\right|\right) \int_{M_{t}}|\nabla v|^{2} d \mu_{t}
$$

and so (20) becomes

$$
\frac{d}{d t} \int_{A} v^{2} d \mu_{t}+c\left(n, M_{0}, k_{1}\right)\left(\int_{A} v^{2 q} d \mu_{t}\right)^{\frac{1}{q}} \leq h(t) \sigma p \int_{A} f_{\sigma}^{p} H^{2} d \mu_{t} .
$$

This is the same inequality as at the corresponding point in [Hu1] except for the function $h(t)$. But using Corollary 5.3,

$$
\frac{d}{d t} \int_{A} v^{2} d \mu_{t}+c\left(n, M_{0}, k_{1}\right)\left(\int_{A} v^{2 q} d \mu_{t}\right)^{\frac{1}{q}} \leq \sigma p S \int_{A} f_{\sigma}^{p} H^{2} d \mu_{t}
$$

and we may proceed again as in [Hu1]. Integrating over $[0, t], t \in[0, T)$,

$$
\begin{aligned}
\int_{A(k)_{t}} v^{2} d \mu_{t}+c\left(n, M_{0}, k_{1}\right) \int_{0}^{t}\left(\int_{A(k)_{t}} v^{2 q} d \mu_{t}\right)^{\frac{1}{q}} d t & \\
& \leq \sigma p S \int_{0}^{t} \int_{A(k)_{t}} f_{\sigma}^{p} H^{2} d \mu_{t} d t .
\end{aligned}
$$

Since $v^{2 q}$ and $f_{\sigma}^{p} H^{2}$ are nonnegative, (22) shows for any $t \in[0, T)$,

$$
\sup _{t \in[0, T)} \int_{A(k)_{t}} v^{2} d \mu_{t} \leq \sigma p S \int_{0}^{T} \int_{A(k)_{t}} f_{\sigma}^{p} H^{2} d \mu_{t} d t
$$

and

$$
c\left(n, M_{0}, k_{1}\right) \int_{0}^{T}\left(\int_{A(k)_{t}} v^{2 q} d \mu_{t}\right)^{\frac{1}{q}} d t \leq \sigma p S \int_{0}^{T} \int_{A(k)_{t}} f_{\sigma}^{p} H^{2} d \mu_{t} d t
$$


Let $q_{0}$ be such that $1<q_{0}<q=\frac{n}{n-1}$. Lemma 6.10 gives

$$
\int_{A(k)_{t}} v^{2 q_{0}} d \mu_{t} \leq\left(\int_{A(k)_{t}} v^{2 q} d \mu_{t}\right)^{\frac{1}{q}}\left(\int_{A(k)_{t}} v^{2} d \mu_{t}\right)^{\left(q_{0}-1\right)}
$$

Hence

$$
\int_{0}^{T} \int_{A(k)_{t}} v^{2 q_{0}} d \mu_{t} d t \leq\left\{\sup _{t \in[0, T)} \int_{A(k)_{t}} v^{2} d \mu_{t}\right\}^{\left(q_{0}-1\right)} \int_{0}^{T}\left(\int_{A(k)_{t}} v^{2 q} d \mu_{t}\right)^{\frac{1}{q}} d t .
$$

Now using inequalities (23) and (24),

$$
\int_{0}^{T} \int_{A(k)_{t}} v^{2 q_{0}} d \mu_{t} d t \leq\left\{c\left(n, M_{0}\right) \sigma p \int_{0}^{T} \int_{A(k)_{t}} f_{\sigma}^{p} H^{2} d \mu_{t} d t\right\}^{q_{0}} .
$$

Exactly as in [Hu1], we then find, using the Hölder inequality, Corollary 6.7 and Lemma 6.11, that

$$
f_{\sigma} \leq k_{1}+D
$$

where $D^{p}=2^{\frac{\gamma}{\gamma-1}} C_{2}\left|A\left(k_{1}\right)\right|_{T}^{\gamma-1},|A(k)|_{T}:=\int_{0}^{T} \int_{A(k)_{t}} d \mu_{t} d t, \gamma=\gamma(n)>1$ and $k_{1}$ and $C_{2}$ depend only on $n$ and $M_{0}$. So for $f_{\sigma}$ to be bounded, it remains to check that $|A(k)|_{T}$ is bounded independently of $T$. We do this as follows. ${ }^{2}$ From inequality (18), since $\varepsilon^{2} p \geq 100$,

$$
\frac{d}{d t} \int_{M_{t}} f_{\sigma}^{p} d \mu_{t} \leq-\int_{M_{t}} f_{\sigma}^{p} H d \mu_{t}+(\sigma p-1) h \int_{M_{t}} f_{\sigma}^{p} H^{2} d \mu_{t}
$$

Choose $\sigma$ small enough such that $\sigma p \leq 1$, so

$$
\frac{d}{d t} \int_{M_{t}} f_{\sigma}^{p} d \mu_{t}+\int_{M_{t}} f_{\sigma}^{p} H d \mu_{t} \leq 0 .
$$

Integrating over time $t$, we obtain

$$
\left.\int_{M_{t}} f_{\sigma}^{p} d \mu_{t}\right|_{t=T}+\int_{0}^{T} \int_{M_{t}} f_{\sigma}^{p} H d \mu_{t} d t \leq\left.\int_{M_{t}} f_{\sigma}^{p} d \mu_{t}\right|_{t=0}=c_{+}\left(M_{0}\right)<\infty .
$$

Now on $A(k)_{t}, k \leq f_{\sigma} \leq H^{\sigma}$ by Lemma 1.4, ii), so $k^{\frac{1}{\sigma}} \leq H$. Therefore, on $A(k)_{t}$,

$$
1 \leq \frac{f_{\sigma}^{p}}{k^{p}} \leq \frac{f_{\sigma}^{p}}{k^{p}} \cdot \frac{H}{k^{\frac{1}{\sigma}}}=\frac{1}{k^{\left(p+\frac{1}{\sigma}\right)}} H f_{\sigma}^{p} .
$$

Finally

$$
|A(k)|_{T} \leq \frac{1}{k^{\left(p+\frac{1}{\sigma}\right)}} \int_{0}^{T} \int_{M_{t}} H f_{\sigma}^{p} d \mu_{t} d t \leq \frac{c_{+}\left(M_{0}\right)}{k^{\left(p+\frac{1}{\sigma}\right)}} \text { by }(25) .
$$

This bound depends only on $n$ and $M_{0}$ and is, in particular, independent of $T$. This completes the proof of Theorem 6.1.

\footnotetext{
${ }^{2}$ I would like to thank Professor Gerhard Huisken for supplying a similar argument for the volume preserving mean curvature flow, which did not appear in [Hu2].
} 


\section{Estimate for the gradient of the mean curvature.}

TheOREM 7.1. For all $\eta>0$ there is a constant $C_{A}=C_{A}\left(n, M_{0}, \eta\right)<\infty$ such that, for all $t \in[0, T)$,

$$
|\nabla H|^{2} \leq \eta H^{4}+C_{A}
$$

This is the same result as in [Hu1] for the original mean curvature flow. In [Hu2], Huisken obtained for the volume preserving mean curvature flow the slightly weaker result

$$
|\nabla H|^{2} \leq \eta H_{T}^{4}+C\left(n, M_{0}, \eta\right)
$$

where $H_{T}=\max _{t \in[0, T)} \max _{x \in M_{t}} H(x, t)$. However, using the Aleksandrov inequality for mixed volumes, we obtain in Section 9 the stronger estimate for the volume preserving mean curvature flow as well.

Using Corollary 4.5, ii) and Lemma 1.9, we easily compute:

LEMMA 7.2. For $t \in[0, T)$,

$$
\begin{aligned}
\frac{\partial}{\partial t}|\nabla H|^{2}=h \Delta|\nabla H|^{2} & -2 h\left|\nabla^{2} H\right|^{2}-2 h\left\langle h_{i j}, \nabla_{i} H \nabla_{j} H\right\rangle+2 h|A|^{2}|\nabla H|^{2} \\
& +2 h\left\langle\nabla_{i} H \nabla_{j} H, h_{i m} h_{j}^{m}\right\rangle-2(1-h H)\left\langle\nabla_{i}|A|^{2}, \nabla_{i} H^{\prime}\right\rangle .
\end{aligned}
$$

Then using the Cauchy-Schwarz inequality, Corollaries 2.2 and 2.3 and Lemmas 1.4 , ii) and 1.5 , we have

Corollary 7.3. For $t \in[0, T)$,

$$
\frac{\partial}{\partial t}|\nabla H|^{2} \leq h \Delta|\nabla H|^{2}+2 n h H|\nabla A|^{2}+4 n H|\nabla A|^{2}+8 n h H^{2}|\nabla A|^{2} .
$$

The following inequalities, similar to those in [Hu2], are easily obtained using Theorem 6.1 and the Cauchy-Schwarz and Young's inequalities.

LEMMA 7.4. For $t \in[0, T)$,

i) $\left|\left\langle\nabla_{i} H, \nabla_{i}\left(|A|^{2}-\frac{1}{n} H^{2}\right)\right\rangle\right| \leq \frac{1}{2}\left\{\frac{(n-1)}{3 n} H+c_{1}\left(n, M_{0}\right)\right\}|\nabla A|^{2}$,

ii) $H\left|\left\langle\nabla_{i} H, \nabla_{i}\left(|A|^{2}-\frac{1}{n} H^{2}\right)\right\rangle\right| \leq \frac{1}{4}\left\{\frac{(n-1)}{3 n} H^{2}+c_{2}\left(n, M_{0}\right)\right\}|\nabla A|^{2}$.

Using Lemma 7.4 and Corollaries 4.6, ii) and 4.5, we obtain

LEMMA 7.5. For $t \in[0, T)$,

i) $\frac{\partial}{\partial t}\left(|A|^{2}-\frac{1}{n} H^{2}\right)$

$$
\leq h\left\{\Delta\left(|A|^{2}-\frac{1}{n} H^{2}\right)-\frac{4(n-1)}{3 n}|\nabla A|^{2}+2|A|^{2}\left(|A|^{2}-\frac{1}{n} H^{2}\right)\right\},
$$

ii) $\frac{\partial}{\partial t}\left\{H\left(|A|^{2}-\frac{1}{n} H^{2}\right)\right\}$

$$
\begin{aligned}
& \leq h\left[\Delta\left\{H\left(|A|^{2}-\frac{1}{n} H^{2}\right)\right\}-\frac{(n-1)}{3 n} H|\nabla A|^{2}+c_{1}|\nabla A|^{2}\right] \\
& +(3 h H-1)|A|^{2}\left(|A|^{2}-\frac{1}{n} H^{2}\right),
\end{aligned}
$$

iii) $\frac{\partial}{\partial t}\left\{H^{2}\left(|A|^{2}-\frac{1}{n} H^{2}\right)\right\}$

$$
\begin{aligned}
& \leq h\left[\Delta\left\{H^{2}\left(|A|^{2}-\frac{1}{n} H^{2}\right)\right\}-\frac{(n-1)}{3 n} H^{2}|\nabla A|^{2}+c_{2}|\nabla A|^{2}\right] \\
& -2 h|A|^{2}(1-2 h H)|A|^{2}\left(|A|^{2}-\frac{1}{n} H^{2}\right) .
\end{aligned}
$$


Here $c_{1}\left(n, M_{0}\right)$ and $c_{2}\left(n, M_{0}\right)$, are the constants of Lemma 7.4.

Proof of Theorem 7.1. Consider the function

$$
g=|\nabla H|^{2}+\left(N_{1}+N_{2} H+N_{3} H^{2}\right)\left(|A|^{2}-\frac{1}{n} H^{2}\right)
$$

where $N_{1}, N_{2}$ and $N_{3}$ are large constants to be chosen later. In [Hu2], Huisken instead used

$$
\begin{gathered}
g_{1}=\left(H+h_{V}\right) H\left(|A|^{2}-\frac{1}{n} H^{2}\right) \\
g_{2}=\left(1+h_{V}\right)\left(|A|^{2}-\frac{1}{n} H^{2}\right)
\end{gathered}
$$

then

$$
g_{3}=|\nabla H|^{2}+N_{2} g_{1}+N_{2} N_{1} g_{2}
$$

for $N_{1}$ and $N_{2}$ suitably large. By using $g$ we avoid the evolution equation for $h(t)$, but we do need the bounds on $h(t)$ from Section 5 .

We compute using Corollary 7.3 and Lemma 7.5,

$$
\begin{aligned}
\frac{\partial}{\partial t} g \leq & h \Delta g+h\left\{N_{2} c_{1}+N_{3} c_{2}-\frac{4(n-1)}{3 n} N_{1}\right\}|\nabla A|^{2} \\
& +\left\{2 n h+4 n-\frac{(n-1)}{3 n} h N_{2}\right\} H|\nabla A|^{2}+h\left\{8 n-\frac{(n-1)}{3 n} N_{3}\right\} H^{2}|\nabla A|^{2} \\
& +\left\{2 h N_{1}+(3 h H-1) N_{2}+2 H(2 h H-1) N_{3}\right\}|A|^{2}\left(|A|^{2}-\frac{1}{n} H^{2}\right)
\end{aligned}
$$

We estimate the terms above not involving $|\nabla A|^{2}$ using the bounds on $h(t)$, Theorem 6.1 and Young's inequality:

$$
\begin{aligned}
& \left\{2 h N_{1}+(3 h H-1) N_{2}+2 H(2 h H-1) N_{3}\right\}|A|^{2}\left(|A|^{2}-\frac{1}{n} H^{2}\right) \\
& \leq\left(2 S N_{1}+3 S N_{2} H+4 S N_{3} H^{2}\right) C_{0} H^{4-\delta} \leq \eta H^{6}+C_{B}\left(n, M_{0}, \eta\right) H^{2}
\end{aligned}
$$

for all $\eta>0$.

Also, using Corollaries 5.3 and 5.7,

$$
2 n h+4 n-\frac{(n-1)}{3 n} h N_{2} \leq 2 n(S+2)-\frac{(n-1)}{3 n} L N_{2}
$$

so if we take $N_{2} \geq \frac{6 n^{2}(S+2)}{(n-1) L}$, the $H|\nabla A|^{2}$ term in (28) may be neglected, leaving

$$
\begin{aligned}
\frac{\partial}{\partial t} g \leq & h \Delta g+h\left\{N_{2} c_{1}+N_{3} c_{2}-\frac{4(n-1)}{3 n} N_{1}\right\}|\nabla A|^{2} \\
& +h\left\{8 n-\frac{(n-1)}{3 n} N_{3}\right\} H^{2}|\nabla A|^{2}+\eta H^{6}+C_{B}\left(n, M_{0}, \eta\right) H^{2} .
\end{aligned}
$$


We now show by contradiction that for any $\eta>0$ there is a constant $C_{A}\left(n, M_{0}, \eta\right)$ such that, for $0 \leq t<T$,

$$
g<\eta H^{4}+C_{A}
$$

Choose $C_{A}$ large enough that the inequality holds at $t=0$ and then suppose there is a first time $t=t_{0}<T$ where $g=\eta H^{4}+C_{A}$ at some $x_{0} \in M_{t_{0}}$. This point is a local maximum of $g$, so

$$
\begin{aligned}
0 \leq & h\left\{N_{2} c_{1}+N_{3} c_{2}-\frac{4(n-1)}{3 n} N_{1}\right\}|\nabla A|^{2} \\
& +h\left\{8 n-\frac{(n-1)}{3 n} N_{3}\right\} H^{2}|\nabla A|^{2}+\eta H^{6}+C_{B} H^{2} \\
\leq & h\left[\left\{N_{2} c_{1}+N_{3} c_{2}-\frac{4(n-1)}{3 n} N_{1}\right\}+\left\{8 n-\frac{(n-1)}{3 n} N_{3}\right\} H^{2}\right] \frac{1}{n}|\nabla H|^{2} \\
& +\eta H^{6}+C_{B} H^{2}
\end{aligned}
$$

by Lemma 1.5, i), since $N_{1}, N_{2}$ and $N_{3}$ are to be chosen so large that the coefficients of all the $|\nabla A|^{2}$ terms are negative. Now substitute for $|\nabla H|^{2}$ using (27). Hence at $x_{0}$

$$
\begin{aligned}
0 \leq & h\left[\left\{N_{2} c_{1}+N_{3} c_{2}-\frac{4(n-1)}{3 n} N_{1}\right\}+\left\{8 n-\frac{(n-1)}{3 n} N_{3}\right\} H^{2}\right] \frac{1}{n} . \\
& \left\{\eta H^{4}+C_{A}-\left(N_{1}+N_{2} H+N_{3} H^{2}\right)\left(|A|^{2}-\frac{1}{n} H^{2}\right)\right\} \\
& +\eta H^{6}+C_{B} H^{2} .
\end{aligned}
$$

Using Theorem 6.1 and Young's inequality,

$$
\left(N_{1}+N_{2} H+N_{3} H^{2}\right)\left(|A|^{2}-\frac{1}{n} H^{2}\right) \leq \frac{1}{2} \eta H^{4}+\frac{1}{2} C_{A}\left(n, M_{0}, \eta\right)
$$

where we may take a larger $C_{A}\left(n, M_{0}, \eta\right)$ earlier if necessary for inequality (30) to also hold. $C_{A}$ would then also depend upon $N_{1}, N_{2}$ and $N_{3}$, but these will all be chosen depending only on $n, M_{0}$ and $\eta$. Hence

$$
\eta H^{4}+C_{A}-\left(N_{1}+N_{2} H+N_{3} H^{2}\right)\left(|A|^{2}-\frac{1}{n} H^{2}\right) \geq \frac{1}{2} \eta H^{4}+\frac{1}{2} C_{A}
$$

so substituting into (29),

$$
\begin{aligned}
0 \leq & \frac{h}{n}\left[\left\{N_{2} c_{1}+N_{3} c_{2}-\frac{4(n-1)}{3 n} N_{1}\right\}+\left\{8 n-\frac{(n-1)}{3 n} N_{3}\right\} H^{2}\right] . \\
& \left(\frac{1}{2} \eta H^{4}+\frac{1}{2} C_{A}\right)+\eta H^{6}+C_{B} H^{2} \\
= & \frac{h}{n}\left[\frac{\eta}{2}\left\{8 n-\frac{(n-1)}{3 n} N_{3}\right\} H^{6}+\frac{\eta}{2}\left\{N_{2} c_{1}+N_{3} c_{2}-\frac{4(n-1)}{3 n} N_{1}\right\} H^{4}\right] \\
& +\frac{h}{2 n}\left[\left\{8 n-\frac{(n-1)}{3 n} N_{3}\right\} C_{A} H^{2}+\left\{N_{2} c_{1}+N_{3} c_{2}-\frac{4(n-1)}{3 n} N_{1}\right\} C_{A}\right] \\
& +\eta H^{6}+C_{B} H^{2} .
\end{aligned}
$$


Grouping the like powers of $H$ and again using Corollary 5.7 gives

$$
\begin{aligned}
& 0 \leq\left[\frac{L}{2 n} \eta\left\{8 n-\frac{(n-1)}{3 n} N_{3}\right\}+\eta\right] H^{6}+\frac{h}{2 n} \eta\left\{N_{2} c_{1}+N_{3} c_{2}-\frac{4(n-1)}{3 n} N_{1}\right\} H^{4} \\
& +\left[\frac{L}{2 n}\left\{8 n-\frac{(n-1)}{3 n} N_{3}\right\} C_{A}+C_{B}\right] H^{2}+\frac{h}{2 n}\left\{N_{2} c_{1}+N_{3} c_{2}-\frac{4(n-1)}{3 n} N_{1}\right\} C_{A} .
\end{aligned}
$$

Now choose $N_{3}=N_{3}\left(n, M_{0}, \eta\right)$ large enough such that both

$$
\frac{L}{2 n}\left\{8 n-\frac{(n-1)}{3 n} N_{3}\right\} C_{A}\left(n, M_{0}, \eta\right)+C_{B}\left(n, M_{0}, \eta\right) \leq 0
$$

and

$$
\frac{L}{2 n} \eta\left\{8 n-\frac{(n-1)}{3 n} N_{3}\right\}+\eta \leq 0 .
$$

Then choose $N_{1}=N_{1}\left(n, M_{0}, \eta\right)$ large enough that

$$
N_{2} c_{1}+N_{3} c_{2}-\frac{4(n-1)}{3 n} N_{1}<0 .
$$

This gives a contradiction in (31). Thus on $0 \leq t<T$,

$$
g=|\nabla H|^{2}+\left(N_{1}+N_{2} H+N_{3} H^{2}\right)\left(|A|^{2}-\frac{1}{n} H^{2}\right) \leq \eta H^{4}+C_{A}\left(n, M_{0}, \eta\right)
$$

and so

$$
|\nabla H|^{2} \leq \eta H^{4}+C_{A}\left(n, M_{0}, \eta\right) .
$$

This completes the proof of Theorem 7.1.

As in [Ha], [Hu1] and [Hu2] we next use

Theorem 7.6 (Myer's Theorem). If $R_{i j} \geq(n-1) K g_{i j}$ along a geo-desic of length at least $\pi K^{-\frac{1}{2}}$ on $M$, then the geodesic has conjugate points.

Similarly as in [Hu1] and [Hu2], to use Myer's theorem we need

LEMMA 7.7. The inequality

$$
R_{i j} \geq(n-1) \varepsilon^{2} H^{2} g_{i j}
$$

is preserved for $t \in[0, T)$, where $\varepsilon>0$ is the constant of Lemma 2.5.

The proof of this lemma is a straightforward contraction of the Gauss equations followed by two applications of the inequality of Theorem 2.5.

COROLlaRY 7.8. Under the surface area preserving mean curvature flow, the mean curvature $H$ of $M_{t}$ is uniformly bounded above for $t \in[0, T)$.

The proof of this corollary is almost identical to the corresponding proof in [Hu2], using Theorem 7.1, Theorem 2.5 and Myer's theorem to show that if $H$ were not uniformly bounded, then all principal curvatures of $M_{t}$ would tend to infinity everywhere. In [Hu2] this contradicts the constant volume property, while here this would contradict the constant surface area property, Lemma 2.6 . 
8. Estimates for higher derivatives of curvature and long time existence. Following closely the technique of Huisken in [Hu2], inspired by Hamilton in [Ha], and also using the bounds on $h(t)$ from Section 5 , it is straightforward to derive the following result.

THEOREM 8.1. For $t \in[0, T)$ and each $m \in \mathbb{N}$,

$$
\left|\nabla^{m} A\right|^{2} \leq C_{m}
$$

uniformly on $M_{t}$, where $C_{m}=C_{m}\left(n, M_{0}\right)$.

Here $\nabla^{m} T$ denotes the $m$ th iterated covariant derivative of a tensor $T$. Since the uniform bounds on $\left|\nabla^{m} A\right|^{2}$ for all $m$ do not depend on $t$,

Corollary 8.2. We may take $T=\infty$.

Exponential convergence to the sphere follows as in [HuI], using a standard interpolation argument and the Sobolev inequality. In particular, $|A|^{2}-\frac{1}{n} H^{2}$, the speed of the $M_{t}$ 's and all curvature derivatives decay exponentially to zero. Hamilton's theorem for uniformly equivalent metrics, from [Ha], can be used to show that the metrics $g_{i j}(\cdot, t)$ converge uniformly to a smooth, positive definite metric $g_{i j}(\cdot, \infty)$ as $t \rightarrow \infty$. Since $|A|^{2}-\frac{1}{n} H^{2} \rightarrow 0, g_{i j}(\cdot, \infty)$ is the metric of a sphere. By Lemma 2.6 this sphere has surface area equal to $\left|M_{0}\right|$. This completes the proof of Theorem 2.8.

9. Alternative $|\nabla H|$ bound for the volume preserving mean curvature flow. The volume preserving mean curvature flow, with flow equation

$$
\frac{\partial}{\partial t} F(x, t)=\left\{h_{V}(t)-H(x, t)\right\} \nu(x, t)
$$

where

$$
h_{V}(t)=\frac{\int_{M_{t}} H d \mu_{t}}{\left|M_{t}\right|},
$$

is shown in [Hu2] to preserve the volume $V$ enclosed by $M_{t}$, while the surface area $\left|M_{t}\right|$ is nonincreasing. Furthermore, the flow preserves strict convexity for a short time, so using the Aleksandrov-Fenchel inequality for mixed volumes to estimate $\int_{M_{t}} H d \mu_{t}$, we can bound $|\nabla H|$ in a similar way as in Section 5. This simplifies the argument in [Hu2] and also avoids computing the evolution equation for $h_{V}(t)$.

By the Favard inequality, (38),

$$
\int_{M_{t}} H d \mu_{t} \leq c(n) \frac{\left|M_{t}\right|^{2}}{V} .
$$

Therefore, recalling (33),

$$
h_{V}(t) \leq c(n) \frac{\left|M_{t}\right|}{V} \leq c(n) \frac{\left|M_{0}\right|}{V}:=S_{V}\left(n, M_{0}\right)
$$

since $\left|M_{t}\right|$ is nonincreasing under the flow. It was shown in [Hu2] that

$$
\begin{gathered}
\frac{\partial}{\partial t}|\nabla H|^{2} \leq \Delta|\nabla H|^{2}+8 n H\left(H+h_{V}\right)|\nabla A|^{2}, \\
\frac{\partial}{\partial t} H=\Delta H+\left(H-h_{V}\right)|A|^{2}
\end{gathered}
$$


and

$$
\frac{\partial}{\partial t}\left(|A|^{2}-\frac{1}{n} H^{2}\right) \leq \Delta\left(|A|^{2}-\frac{1}{n} H^{2}\right)-\frac{4(n-1)}{3 n}|\nabla A|^{2}+2|A|^{2}\left(|A|^{2}-\frac{1}{n} H^{2}\right) .
$$

Using these we compute that the function

$$
g=|\nabla H|^{2}+\left\{N_{1}+N_{2} H+N_{3} H^{2}\right\}\left(|A|^{2}-\frac{1}{n} H^{2}\right)
$$

where $N_{1}, N_{2}$ and $N_{3}$ are large constants to be chosen later, satisfies

$$
\begin{aligned}
\frac{\partial}{\partial t} g \leq & \Delta g+\left\{N_{2} c_{1}+N_{3} c_{2}-\frac{4(n-1)}{3 n} N_{1}\right\}|\nabla A|^{2} \\
& +\left\{8 n h-\frac{(n-1)}{3 n} N_{2}\right\} H|\nabla A|^{2}+\left\{8 n-\frac{(n-1)}{3 n} N_{3}\right\} H^{2}|\nabla A|^{2} \\
& +\left\{2 N_{1}+3 H N_{2}+4 H^{2} N_{3}\right\}|A|^{2}\left(|A|^{2}-\frac{1}{n} H^{2}\right)
\end{aligned}
$$

where $c_{1}$ and $c_{2}$, constants depending only on $n$ and $M_{0}$, arise in the same way as in Section 7. It follows similar as in Section 7, using (34) and the equivalent pinching estimate from [Hu2] to Theorem 6.1, that for all $\eta>0$,

$$
|\nabla H|^{2} \leq \eta H^{4}+C\left(n, M_{0}, \eta\right) \text {. }
$$

A lower positive bound on $h_{V}(t)$ is not needed here.

Appendix A. Curvature integral estimates using mixed volumes and applications. As in [BZ], we write the $m$-th mixed volume of a convex region $E_{t}$ as

$$
V_{m}\left(E_{t}\right)=\frac{1}{(n+1)\left(\begin{array}{c}
n \\
m
\end{array}\right)} \int_{M_{t}} \sum_{j} \kappa_{j_{1}} \cdots \kappa_{j_{n-m}} d \mu_{t}
$$

where $E_{t}$ is the solid $(n+1)$-dimensional region bounded by $M_{t}, M_{t}$ has principal curvatures $\kappa_{1}, \ldots, \kappa_{n}$ and the sum is taken over the $\left(\begin{array}{c}n \\ n-m\end{array}\right)$ various finite sequences $j=\left(j_{1}, \ldots, j_{n-m}\right), 1 \leq j_{i} \leq n$.

The $(n+1)$-th mixed volume is

$$
V_{n+1}\left(E_{t}\right)=\operatorname{Vol}\left(E_{t}\right) .
$$

Two consequences of the Aleksandrov-Fenchel inequality for mixed volumes are the Favard inequality,

$$
V_{i}^{2}\left(E_{t}\right) \geq V_{i-1}\left(E_{t}\right) V_{i+1}\left(E_{t}\right)
$$

which holds for any $i=1, \ldots, n$ and

$$
V_{i}^{n+1}\left(E_{t}\right) \geq \omega_{n+1}^{n+1-i} V^{i}\left(E_{t}\right)=\omega_{n+1}^{n+1-i} \operatorname{Vol}^{i}\left(E_{t}\right)
$$

where $\omega_{n+1}$ is the volume of the $(n+1)$-dimensional unit ball. Remembering Lemma 2.7, for the surface area preserving mean curvature flow this becomes

$$
V_{i}^{n+1}\left(E_{t}\right) \geq \omega_{n+1}^{n+1-i} \operatorname{Vol}^{i}\left(E_{0}\right) .
$$


For an upper bound on $\int_{M_{t}} \sum_{i<j} \kappa_{i} \kappa_{j} d \mu_{t}$, note from (36),

$$
\begin{gathered}
V_{n-2}\left(E_{t}\right)=\frac{2}{(n+1) n(n-1)} \int_{M_{t}} \sum_{j} \kappa_{j_{1}} \kappa_{j_{2}} d \mu_{t}, \\
V_{n-1}\left(E_{t}\right)=\frac{1}{(n+1) n} \int_{M_{t}} H d \mu_{t}
\end{gathered}
$$

and using also Lemma 2.6,

$$
V_{n}\left(E_{t}\right)=\frac{1}{n+1}\left|M_{t}\right|=\frac{1}{n+1}\left|M_{0}\right|
$$

Now from (38),

$$
V_{n-2}\left(E_{t}\right) \leq \frac{V_{n-1}^{2}\left(E_{t}\right)}{V_{n}\left(E_{t}\right)}
$$

so substituting in (41), (42) and (43) gives

$$
\int_{M_{t}} \sum_{i<j} \kappa_{i} \kappa_{j} d \mu_{t} \leq \frac{n-1}{2 n\left|M_{0}\right|}\left(\int_{M_{t}} H d \mu_{t}\right)^{2} .
$$

Using (42), (43) and (37) in (38) with $i=n$ yields

$$
\int_{M_{t}} H d \mu_{t} \leq \frac{n}{(n+1)} \cdot \frac{\left|M_{0}\right|^{2}}{\operatorname{Vol}\left(E_{t}\right)} \leq \frac{n}{(n+1)} \cdot \frac{\left|M_{0}\right|^{2}}{\operatorname{Vol}\left(E_{0}\right)}
$$

by Lemma 2.7. So we have an upper bound on $\int_{M_{t}} H d \mu_{t}$ without using the diameter bound. (For this we could instead have used the Cauchy-Schwarz inequality, Lemma 2.6 and Corollary 5.6, again not needing the diameter bound.)

Substituting this into (44) gives

$$
\int_{M_{t}} \sum_{i<j} \kappa_{i} \kappa_{j} d \mu_{t} \leq \frac{(n-1) n\left|M_{0}\right|^{3}}{2(n+1)^{2} \operatorname{Vol}\left(E_{0}\right)}=: U\left(n, M_{0}\right),
$$

which is the bound required in the proof of Corollary 5.6.

We can also obtain a lower bound on $\int_{M_{t}} H d \mu_{t}$, avoiding the diameter estimate. Setting $i=n-1$ in (39) and recalling (42),

$$
\left\{\frac{1}{(n+1) n} \int_{M_{t}} H d \mu_{t}\right\}^{n+1} \geq \omega_{n+1}^{2} \operatorname{Vol}^{n-1}\left(E_{0}\right)
$$

yielding the desired positive lower bound. Corollaries 5.2 and 5.3, the upper bound on $h$, then follow without the diameter estimate.

The lower bound on $\int_{M_{t}} H d \mu_{t}$ also combines with the upper bound on $\int_{M_{t}} H^{2} d \mu_{t}$ of Corollary 5.6 to yield the lower bound on $h$, Corollary 5.7, again without the diameter estimate. 


\section{REFERENCES}

[An1] B. ANDREws, Evolving convex hypersurfaces, PhD thesis, Australian National University, 1993.

[BZ] Y. Burago, V. A. ZAlgaller, Geometric Inequalities, Springer-Verlag, 1988.

[CG] B. Chow, R. Gulliver, Aleksandrov reflection and geometric evolution equations I: The $n$-sphere and n-ball, Calc. Var., 4 (1996), pp. 249-264.

[Ha] R. S. HAMILTON, Three-manifolds with positive Ricci curvature, J. Differential Geometry, 17 (1982), pp. 255-306.

[Hu1] G. HUISKEN, Flow by mean curvature of convex surfaces into spheres, J. Differential Geometry, 20 (1984), pp. 237-266.

[Hu2] G. HUISKEN, The volume preserving mean curvature flow, J. reine angew. Math., 382 (1987), pp. 35-48.

[Hu3] G. HuIsKen, Asymptotic behaviour for singularities of the mean curvature flow, J. Differential Geometry, 31 (1990), pp. 285-299.

[L] S. R. LAY, Convex sets and their applications, John Wiley and Sons, 1982.

[M] J. A. MCCoY, The mixed volume preserving mean curvature flow, preprint.

[MS] J. H. MiChAEL, L. M. Simon, Sobolev and mean value inequalities on generalized submanifolds of $\mathbb{R}^{n}$, Comm. Pure Appl. Math., 26 (1973), pp. 361-379.

[P] D. M. PIHAN, A length preserving geometric heat flow for curves, $\mathrm{PhD}$ thesis, University of Melbourne (1998).

[Sc] R. SchNeIDER, Convex bodies: The Brunn-Minkowski theory, Cambridge University Press, 1993.

[St] G. Stampacchia, Equations elliptiques au second ordre à coéefficients discontinues, Sém. Math. Sup. 16, Les Presses de l'Université de Montreal, Montreal, 1966.

[U] J. I. E. URBAS, An expansion of convex hypersurfaces, J. Differential Geometry, 33 (1991), pp. 91-125.

[W] T. J. Willmore, An. Sti. Univ. "Al. I. Cuza" Iasi Sect. I a Mat., 11B (1965), pp. 493-496 [M. R., 34, No. 1940]. 